Polymer Journal, Vol. 39, No. 1, pp. 41-47 (2007)

(C) 2006 The Society of Polymer Science, Japan

\title{
Electroless Copper Plating onto Polyimide Using Polymer Nanosheet as a Nano-Adhesive
}

\author{
Jun Matsui, ${ }^{1,2, \dagger}$ Kosuke Kubota, ${ }^{1}$ Yuko Kado, ${ }^{1}$ and Tokuji MiYashita ${ }^{1, \dagger}$ \\ ${ }^{1}$ Institute of Multidisciplinary Research for Advanced Materials, Tohoku University, \\ Katahira 2-1-1, Aoba-ku, Sendai 980-8577, Japan \\ ${ }^{2}$ Precursory Research for Embryonic Science and Technology (PRESTO), \\ Japan Science and Technology Agency, 4-1-8 Honcho, Kawaguchi 332-0012
}

(Received August 24, 2006; Accepted October 18, 2006; Published November 24, 2006)

\begin{abstract}
In this paper, we describe a technique to prepare a copper layer on polyimide film using a polymer nanosheet as a nano-adhesion. We employ two kinds of functional polymer nanosheets: one works as an adhesion layer and the other works as a template layer to adsorb gold nanoparticles, which works as a catalyst for the electroless plating. The photoreactive polymer nanosheet was used to increase the adhesion force between the copper layer and polyimide. Furthermore, the cationic polymer nanosheets were used to adsorb a gold catalyst for electroless copper plating. Applying the technique, electroless plated copper was attached strongly onto polyimide film. Micrometer copper lines were fabricated by photopatterning the polymer nanosheets. The process using polymer nanosheets as an adhesive required no surface modification of polyimide substrate and enabled microscale copper line fabrication without discharging harmful waste. Consequently, the technique is useful for next-generation flexible printed board fabrication. [doi:10.1295/polymj.PJ2006099]

KEY WORDS Flexible Printed Circuit Boards / Polymer Nanosheet / Electroless Copper Plating / Nano-Adhesive /
\end{abstract}

Flexible printed circuit boards (FPCs) are widely used in portable electric devices such as cell phones and digital video cameras because of the flexibility, light wight and high mechanical stability. ${ }^{1}$ For construction of FPCs, polyimides (PIs) are used mostly as substrate because of their high chemical and thermal stability. ${ }^{2}$ Copper is used for the wiring metal because of its high conductivity. To fabricate copper wiring layers onto dielectric polyimide substrates, electroless deposition of copper metal onto polyimide films has become a frequently adopted industrial processes for metallization because of its simplicity and low-cost fabrication. However, the adhesion between pristine PI surface and electroless deposited copper film is too weak for practical applications. Therefore, intense research has examined improvement in the adhesion of plated copper film onto PI surfaces. Most conventional methods to achieve sufficient adhesion between metal layer and PI include physical or chemical etching of the PI surface. ${ }^{3-7}$ However, the treatment increases the surface roughness (typically several micrometers in surface roughness). For that reason it is not applicable for FPCs for microelectronics, which requires a circuit of a few micrometer-scale pattern size. Recently, several methods have been reported to achieve strong adhesive force between copper film and polyimide with micrometer line and scale without creating micrometer-scale surface roughness. For example, Neoh et al. ${ }^{8-14}$ and Inagaki et al. ${ }^{15-19}$ modified polyimide by graft polymerization of vinylimidazole or vinylpyridine. The plated copper layer interacts with the nitrogen group of the graft polymer, which creates a strong adhesive force between the PI and the copper layer. However, the PI surface must be activated using Ar plasma process, which is an expensive and high-energy consumptive process. On the other hand, Akamatsu et al. ${ }^{20-23}$ and Baum et al. ${ }^{24}$ used a base hydrolysis reaction of polyimide to achieve a strong adhesion force with a copper layer. The carboxylic group generated by the hydrolysis reaction formed an ionic complex with copper ion. The ionic complex acts as an anchor to electrolessly deposited copper films. However, there is a possibility of thermal reimidation reaction by Joul heat. Moreover, the ionic complex has a high dielectric constant, which increases the resistance-capacitance (RC) delay.

So far, reported technique to enhance adhesion between copper and PI require chemical modification of PI surface. The modification processes change the PI surface properties and might inadvertently etch the PI surface. Therefore, a new adhesion technique that requires no chemical modification process would be useful for fabrications of the submicrometer line FPCs.

${ }^{\dagger}$ To whom correspondence should be addressed (Tel: +81-22-217-5639, Fax: +81-22-217-5639, E-mail: jun_m@ tagen.tohoku.ac.jp, Tel: +81-22217-5637, Fax: +81-22-217-5642, E-mail: miya@tagen.tohoku.ac.jp). 


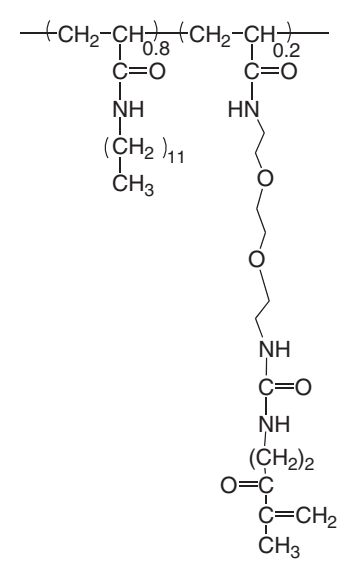

pDDA-M

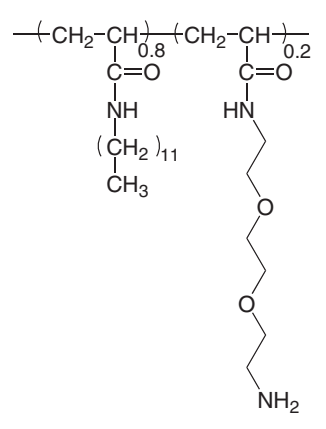

pDDA-DADOO

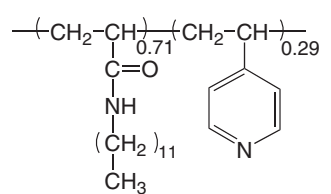

$\mathrm{CH}_{3}$

Figure 1. Chemical structures of polymers used in the study.

In addition to the requirement of strong adhesion force between PI and copper film, the copper film must be patterned in a micrometer range to create circuits. One conventional method is photolithography using organic photoresist materials. However, the photolithography technique discharges many harmful chemical wastes during the development. On the point of green chemistry, a more environmental friendly technique to create a micrometer pattern is necessary for the feature FPC fabrication.

This paper describes a new method to enhance the adhesion strength between electrolessly plated copper film and PI. We apply polymer nanosheets as a nanoadhesive to attach copper lines strongly onto PI. Polymer nanosheets composed of poly $(N$-alkylacrylamide)s are molecular level smooth polymer films with $1-2 \mathrm{~nm}$ thickness. They were prepared using the Langmuir-Blodgett (LB) technique. Moreover, using functional molecules as comonomers, several functional polymer nanosheets were synthesized. ${ }^{25-30}$ The functional polymer nanosheets were applied to develop ultrathin devices. ${ }^{31-33}$ For example, photoreactive polymer nanosheets, which have a vinyl group in the side chains, were applied to photoresist materials. ${ }^{28-30}$ On the other hand, cationic polymer nanosheets with amino or pyridine units were used as a template to assemble gold nanoparticles. ${ }^{34,35} \mathrm{In}$ this study, we carried out a novel technique for electroless deposition of copper film onto PI using polymer nanosheet assemblies. We employ a photoreactive polymer nanosheet as an adhesive layer between PI film and copper layer and cationic template polymer nanosheets for building a catalyst nanoparticle. The photoreactive polymer nanosheet, pDDA-M (Figure 1), which has a methacryloyl group in the side chains, increase adhesion strength between copper film and pristine PI substrate by a photo-cross-linking reaction. The polymer nanosheet with amino (pDDA-
DADOO) or pyridine (pDDA-VPy) group (Figure 1) was applied to assemble gold nanoparticles onto the PI substrate covered with pDDA-M. The assembled gold nanoparticles acted as a catalyst for electroless deposition of the copper layer. The electrolessly plated copper layer showed strong adhesion to the PI substrate and high electric conductivity. Moreover, micrometer line and space copper patterns were fabricated using a photolithography technique without development process.

\section{EXPERIMENTAL}

The synthesis of photoreactive polymer, pDDA$\mathrm{M}$, and cationic polymer pDDA-VPy and pDDADADOO, those were used to adsorb a gold nanoparticle (Figure 1) were described previously. ${ }^{28-30,36-38}$ The gold nanopartcile, which acted as a catalyst of copper plating, were synthesized by reduction of $\mathrm{HAuCl}_{4}$ by sodium citrate according to reported procedures. ${ }^{39,40}$ Polyimide $\left(\right.$ Kapton $^{\circledR}$ ), was provided by Nippon Steel Chemical Co., Ltd. It was washed with isopropanol before used. The polymers were dissolved in chloroform $(1 \mathrm{mM})$ and spread onto the water surface. The water was purified using ultrapure water system (CPW101, Advantec). The measurement of $\pi-A$ isotherms and deposition of monolayers were carried out with an automatic Langmuir trough (USI, LB lift controller FSD-51 with a Wilhelmy-type film balance) at $20^{\circ} \mathrm{C}$ and compressed at a rate of $15 \mathrm{~cm}^{2} / \mathrm{min}$.

The procedure to plate copper onto PI films is illustrated in Figure 2. Firstly, several layers of pDDA-M nanosheet were deposited onto Kapton ${ }^{\circledR}$ with the surface pressure of $35 \mathrm{mN} / \mathrm{m}$ using the $\mathrm{LB}$ technique. Subsequently, these layers were illuminated $10 \mathrm{~min}$ with UV light $\left(70 \mathrm{~mW} / \mathrm{cm}^{2}\right)$ through a water filter using a $500 \mathrm{~W}$ high-pressure mercury lamp (Ushio Inc.) to cross-link methacryloyl group in the pDDA-M lay- 


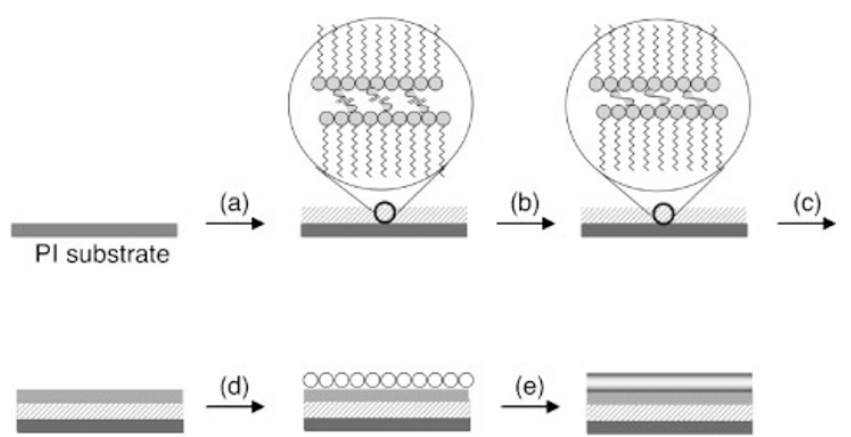

Figure 2. Schematic illustration of the procedure for electroless copper plating onto PI substrate using polymer nanosheets. (a) Several layers of pDDA-M nanosheets were deposited onto PI substrate and then (b) UV light $\left(70 \mathrm{~mW} / \mathrm{cm}^{2}\right)$ was irradiated $10 \mathrm{~min}$ to induce photochemical reaction of methacryloyl group. (c) pDDA-DADOO nanosheets with 2 layers was deposited onto the PI substrate and then (d) immersed into gold nanoparticle aqueous dispersion to adsorb gold nanoparticles. (e) The PI substrate with gold catalyst was immersed into electroless copper plating bath to create copper film.

ers. Subsequently pDDA-VPy or pDDA-DADOO of 2 layers were deposited onto pDDA-M modified PI with the surface pressure of $30 \mathrm{mN} / \mathrm{m}$ at $20^{\circ} \mathrm{C}$. Then they were immersed into gold nanoparticle aqueous dispersion to adsorb gold nanoparticles onto the substrate. Finally the substrate was immersed into an electroless copper plating bath for $40 \mathrm{~s}$ at $70^{\circ} \mathrm{C}$. The gold nanoparticle acted as a catalyst for the plating. The composition of the plating solution was as follows: $0.038 \mathrm{M}$ $\mathrm{CuSO}_{4}, 0.10 \mathrm{M}$ formaldehyde, $0.48 \mathrm{M}$ potassium hydroxide and $0.051 \mathrm{M}$ ethylene diamine tetraacetic acid (EDTA). All chemicals were used as received. The copper deposited PI films were then rinsed thoroughly with copious amount of distilled water. The films surface was observed using optical microscope (Vanox; Olympus Optical Co. Ltd), atomic force microscope (SPA-400, Seiko Instruments Inc.) and scanning electron microscope (SEM) (PHILIPS XL30ESEM). The film thickness was measured using surface profiler (Dektac 3-ST, Veeco). The X-ray diffraction (XRD) measurement was carried out using MAC Science

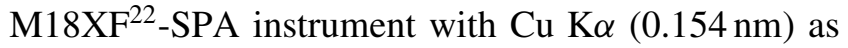
a target. The conductivity of the deposited copper film was measured by four probe point methods with a digital multi meter (Keithley 2400).

\section{RESULTS AND DISCUSSION}

\section{Monolayer Properties of Photofunctional and Cation- ic Polymers}

Monolayer properties of the polymers were investigated by $\pi-A$ isotherm measurements (Figure 3 ). The $\pi-A$ isotherms of pDDA-M, pDDA-DADOO and pDDA-VPy show a steep increase of the surface

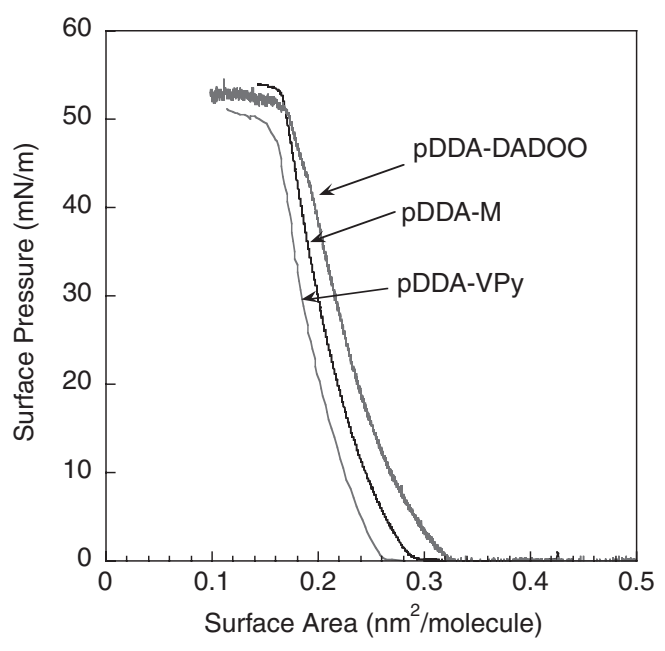

Figure 3. Surface pressure-area isotherms of the polymers.

(a)

(b)

(c)

(d)

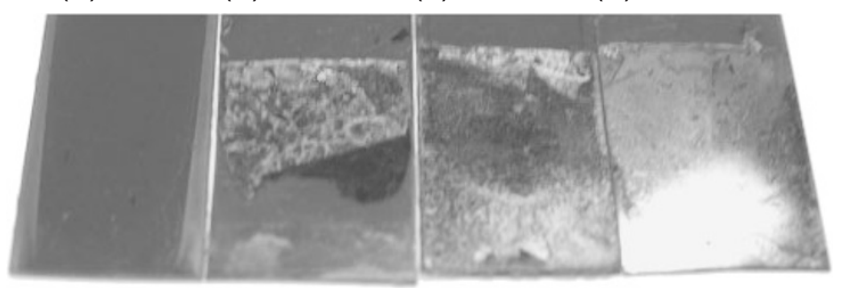

Figure 4. Digital camera images of electrolessly plated copper film onto PI substrate with different numbers of pDDA-M layers. (a) 24 layers, (b) 50 layers, (c) 74 layers, (d) 100 layers. The layer number of pDDA-DADOO was fixed at two layers.

pressure with decreasing surface area, which indicates that the polymers formed a condensed monolayer at the air/water interface. Moreover, the transfer ratios of the polymers were $1.0 \pm 0.1$ in both the downward and upward strokes, resulting in Y-type LB films. Stable film formation of the polymers is attributed to the two-dimensional network between the amide groups of dodecylacrylamide (DDA) as well as amphiphilicity of DDA groups. The polymer nanosheets were deposited onto PI substrate using the LB technique for electroless plating of copper.

\section{Effect of Deposition Number to Electroless Copper Plating}

First, different numbers of pDDA-M monolayer were deposited onto PI substrate to examine effects of the number of deposited layers to electroless copper plating. Figure 4 shows digital camera images of PI substrates after electroless copper plating. At the PI substrate covered with 24 layers of pDDA-M nanosheets, an electrolessly deposited copper film was easily peeled off during the plating process. Moreover, the substrate edges color was turned white after the plating. However, with increasing number of pDDA- 


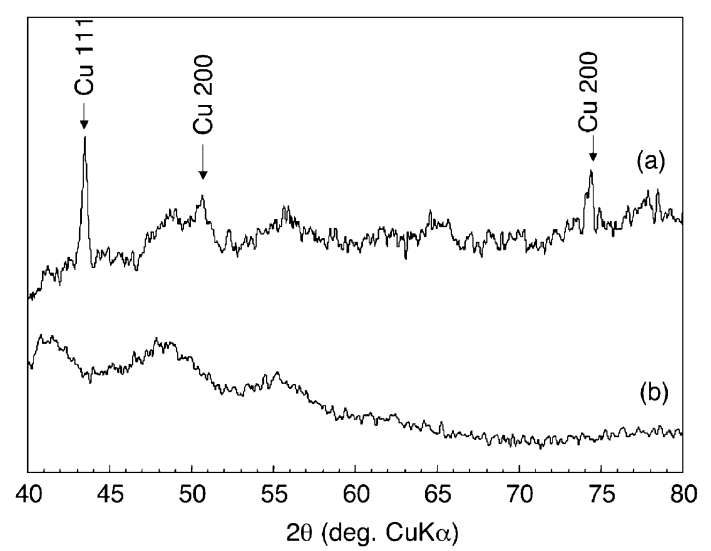

Figure 5. XRD patterns of PI substrates. (a) PI substrate plated with copper using pDDA-M as a nano-adhesive. (b) XRD pattern of PI substrate.

$M$ layers, the plated copper area increased and at the PI substrate covered with 100 layers of pDDA-M, a uniform copper thin film was plated onto PI substrate (Figure 4d). Moreover, the XRD pattern of thin film plated onto the PI substrate covered with 100 layers of pDDA-M clearly indicates that it is a metallic copper film (Figure 5a). ${ }^{41}$

The reasons for the necessity of large number of layers to strongly attach copper film onto PI are following: at the PI substrate covered with small number of layers, the alkali plating solution penetrates into the pDDA-M adhesion layer and reaches to the PI surface. The hydrolysis reaction of PI occurred and the plated copper together with adhesion layer of pDDA-M was peeled off from the PI surface. The white region appeared at the PI covered with a small layer numbers of pDDA-M was attributed to potassium polyamic acid salt formation after the base hydrolysis of the PI surface by highly basic plating solution. However, when 100 layers of pDDA-M were deposited onto PI substrate, pDDA-M layers prevented penetration of the alkali solution and hydrolysis reaction. Therefore a uniform copper film was plated without peeling off the copper film. Plated copper film was peeled off from the PI substrate covered with 100 layers of

(a)

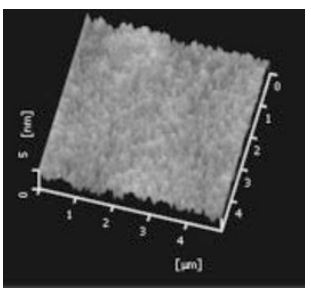

(b)

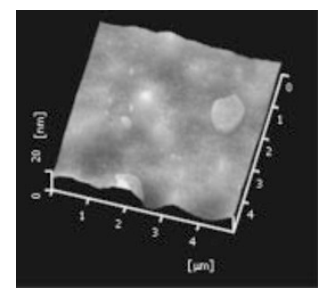

pDDA-M when the irradiation was omitted. Therefore, the photo-crosslinking of methacryloyl groups in pDDA-increased chemical and mechanical strength of pDDA-M. The plated copper thin film onto the PI film covered with 100 layers of cross-linked pDDA$\mathrm{M}$ was highly adhesive; the film readily passes the simple Scotch-tape test. The results indicate that the pDDA-M nanosheets act as a good nano-adhesive to increase the adhesion force between PI and the copper layer.

\section{Surface Profile and Conductivity Measurement of the Plated Copper Film}

The surface of the plated copper film was examined by AFM measurement. The nano-adhesion layer showed a smooth surface with root mean square (RMS) of $2.3 \mathrm{~nm}$ (Figure 6b). After the adsorption of gold nanoparticle, the surface roughness increases to $12.1 \mathrm{~nm}$ (Figure 6c). The gold nanoparticles were adsorbed uniformly onto the nano-adhesive covered PI, ${ }^{34}$ therefore the roughness increase was attributed to the large size $(30 \mathrm{~nm})$ of the gold nanoparticle. The plated copper surface showed a surface with roughness of RMS of $23 \mathrm{~nm}$ (Figure 6d). The roughness was smoother than conventional FPC and enough for fabricating of micrometer copper line. The film resistance was measured to be $270 \mathrm{~m} \Omega$ by a four-probe resistivity measurement. The total thickness of the plated copper layer with the nano-adhesion layer and the thickness of the nano-adhesion layer itself were determined to be $350 \mathrm{~nm}$ and $175 \mathrm{~nm}$ respectively from surface profile measurements. Therefore the thickness of the plated copper layer was calculated to be $175 \mathrm{~nm}$. From the resistance and copper film thickness measurements, specific resistance $(\rho)$ of the plated copper film was calculated to be $5.7 \mu \Omega \mathrm{cm}$ using $\rho=\mathrm{R} \times \mathrm{W} \times \mathrm{t} / \mathrm{L}$, where $\mathrm{R}$ is the film resistance $(\Omega), \mathrm{W}$ is the film width $(0.1 \mathrm{~cm}$ in the present case), $\mathrm{t}$ is the film thickness $\left(180 \times 10^{-7} \mathrm{~cm}\right.$ in the present case) and $\mathrm{L}$ is the measurement length $(0.85 \mathrm{~cm}$ in the present case). The value is three times larger than bulk value $(1.67 \mu \Omega \mathrm{cm})$. The low conduc- (c)

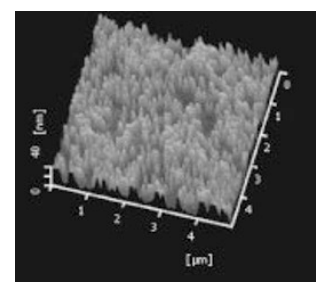

(d)

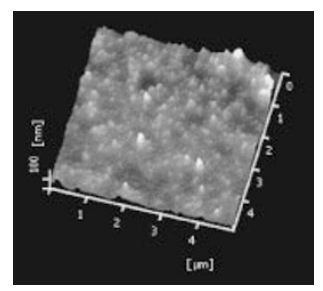

Figure 6. Atomic force microcopy images of PI substrates. (a) pristine PI substrate. (b) PI substrate covered with 100 layers of pDDAM. The film was irradiated 10 min to cross-link methacryloyl groups (c) PI substrate after adsorption of gold nanoparticles. (d) PI substrate after copper electroless plating. 

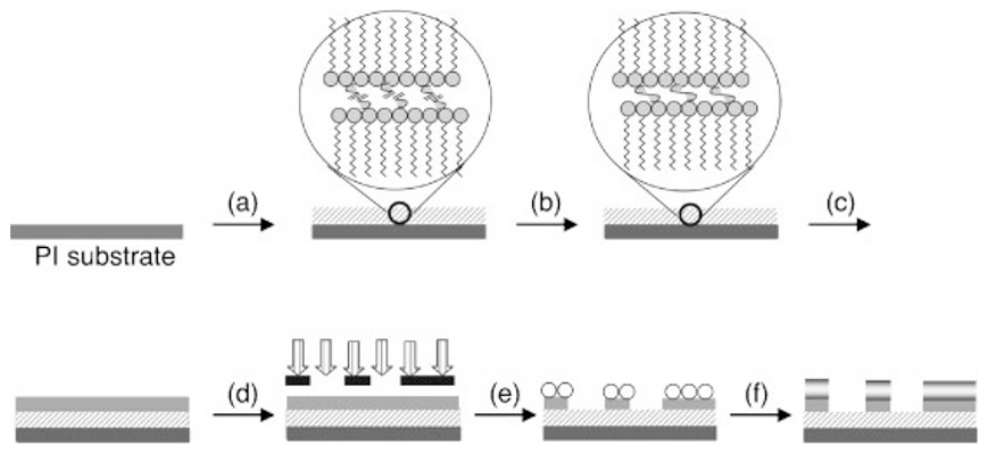

Figure 7. Schematic illustration of the procedure for creating micrometer copper line onto PI film. (a) Hundred layers of pDDA-M nanosheets were deposited onto PI substrate and then (b) UV light $\left(70 \mathrm{~mW} / \mathrm{cm}^{2}\right)$ was irradiated $10 \mathrm{~min}$ to induce photochemical reaction of methacryloyl group. (c) pDDA-VPy nanosheets with 2 layers was deposited onto the PI substrate and then (d) UV light $\left(70 \mathrm{~mW} / \mathrm{cm}^{2}\right)$ was irradiated $10 \mathrm{~min}$ through a photomask to create a fine pattern of pDDA-VPy. (e) The PI substrate with patterned pDDA-VPy was immersed into gold nanoparticle aqueous dispersion to adsorb gold onto unirradiated areas. (f) The PI substrate with gold nanoparticle array was immersed into electroless copper plating bath to create copper line.

(a)

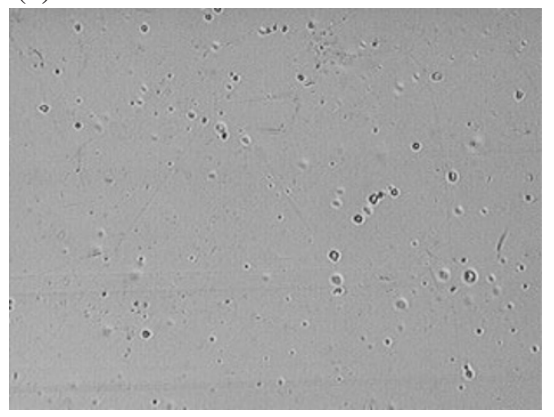

(b)

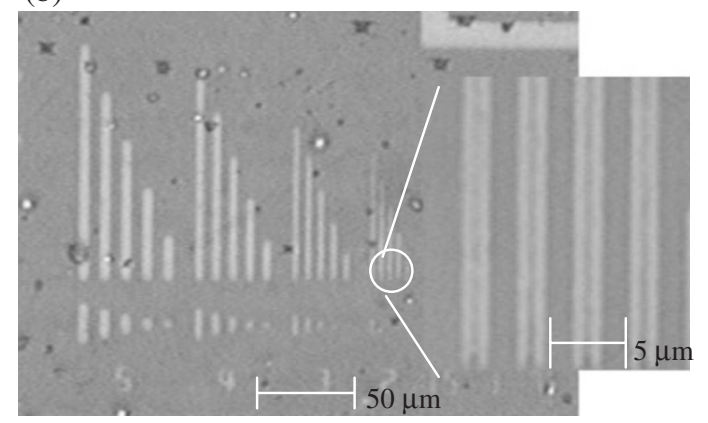

Figure 8. Optical micrograph of (a) pristine PI substrate and (b) gold nanoparticle arrays on the PI substrate. The pDDA-M of 100 layers was used as a nano adhesion and pDDA-VPy was used as a template for gold nanoparticle arrays.

tivity indicates that the plated copper have many grains and grain boundaries. In present, electroless plating condition was not optimized. The surface roughness and the film resistance will be improved with optimizing the electroplating conditions, such as plating temperature, solution concentration and so forth.

\section{Fabrication of Micrometer Copper Pattern Using Photolithography Technique without Development}

In the following section, we demonstrate a patterning of copper film at micrometer scale using photolithography technique. We reported previously that deep UV irradiation on pDDA-VPy through a photomask gives a fine photopattern of pDDA-VPy nanosheets. ${ }^{29}$ Moreover, using the photopatterned nanosheets as a template, gold nanoparticle arrays were constructed. ${ }^{34}$ Therefore for the fabrication of micrometer copper lines onto a PI substrate, pDDA-VPy was used as a catalyst template nanosheets instead of pDDA-DADOO, which had no ability to be photopatterned. Photopatterning of pDDA-VPy required no development process; it can be dry patterned, which means that development processes of patterns are unnecessary. The process was simple, environment friendly and had no effect of film swelling which affected the adhesion strength between PI substrate and copper layer. Figure 7 shows the procedure to create micrometer copper line onto PI substrate using the polymer nanosheets as a nano adhesion. Two layers of pDDA-VPy nanosheets were deposited onto the PI substrate covered with cross-linked pDDA-M of 100 layers to develop micrometer copper lines onto PI substrate. Subsequently the PI substrate was irradiated through a photomask to decompose VPy in the irradiated region using $500 \mathrm{~W}$ high-pressure mercury lamp $\left(70 \mathrm{~mW} / \mathrm{cm}^{2}, 10 \mathrm{~min}\right)$. The PI substrate with patterned pDDA-VPy nanosheets were immersed into a gold nanoparticle solution. The optical microscope and scanning electron microscope image reveal that gold nanoparticles were directed selectively onto the unirradiated potion of pDDA-VPy nanosheets, producing configured gold nanoparticle monolayer patterns with $2.0 \mu \mathrm{m}$ resolution (Figure 8). Then the PI substrate with patterned gold nanoparticle arrays was immersed into an electroless copper plating bath to create micro- 


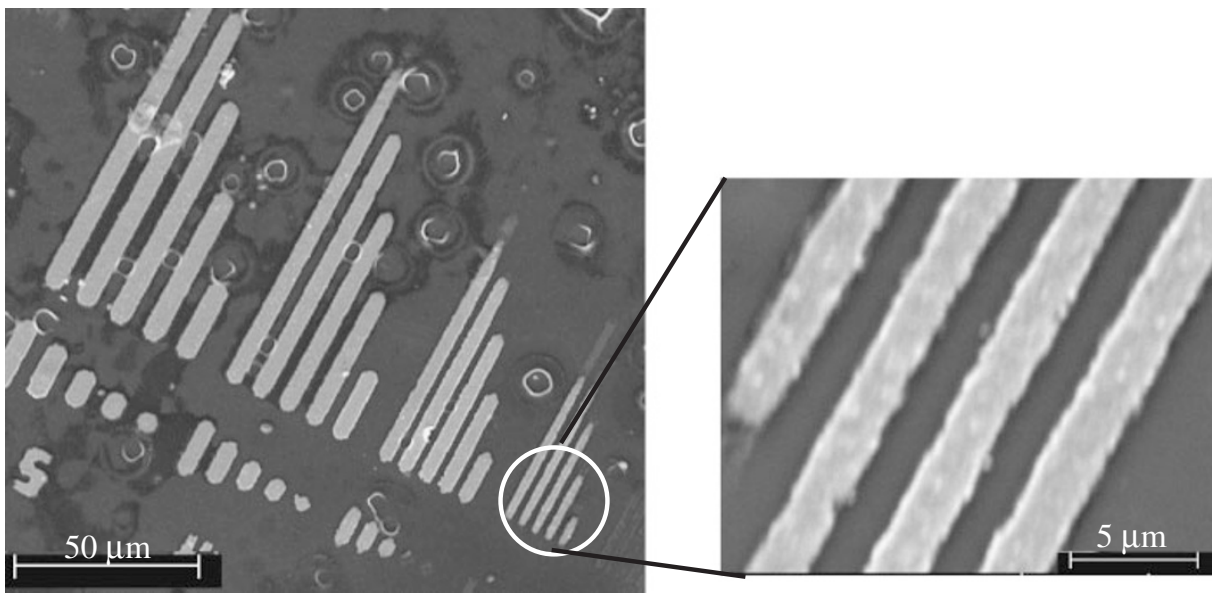

Figure 9. SEM image of electrolessly plated copper lines onto PI substrate. The pDDA-M of 100 layers was used as a nano adhesion and pDDA-VPy was used as a template to produce gold nanoparticle arrays. The cracks owed its origin to PI substrate.

meter copper line. Figure 9 shows an SEM image of the PI substrate after the copper electroless deposition. Fine copper patterns with $2.0 \mu \mathrm{m}$ resolution, which was the same resolution as the gold catalyst arrays, were drawn using the photography technique without development processes.

\section{CONCLUSION}

The photoreactive polymer nanosheet and the gold catalyst template nanosheets were applied to improve the adhesion strength between the electrolessly deposited copper layer and pristine PI substrate. Copper layers were strongly attached onto PI substrate using pDDA-M and pDDA-DADOO as a nano glue to attach the electrolessly deposited copper onto PI substrate. Moreover, with applying pDDA-VPy as a gold template nanosheet, micrometer scale copper lines were fabricated. The process using polymer nanosheets as an adhesion requires no surface modification of PI substrate and enables microscale copper line fabrication without discharging harmful waste. Therefore the process is useful for feature FPC fabrication.

Acknowledgment. This work is partially supported by Grants-in-Aid for Scientific Research Nos. 17105006 and 16750107 from the Ministry of Education, Culture, Sports, Science and Technology, and from The Mitsubishi Foundation. We thank professor H. Nakanishi, Dr. A. Masuhara and Mr. H. Endo for SEM measurements.

\section{REFERENCES}

1. "Polymers for Electronic and Photonic Applications," Academic Press, Boston, MA., 1994.

2. "Polyimides: Fundamental Aspects and Technological Applications,” Marcel Dekker, New York, 1996.
3. K. W. Paik and A. L. Ruoff, J. Adhes. Sci. Technol., 4, 465 (1990).

4. D. Majumdar and R. G. Spahn, J. Adhes. Sci. Technol., 5, 349 (1991).

5. M. Menezes, I. M. Robertson, and H. K. Birnbaum, J. Mater. Res., 14, 4025 (1999).

6. O. Kraft, R. Schwaiger, and P. Wellner, Mater. Sci. Eng., A, 319, 919 (2001).

7. N. Inagaki, S. Tasaka, and A. Onodera, J. Appl. Polym. Sci., 73, 1645 (1999).

8. W. C. Wang, R. H. Vora, E. T. Kang, and K. G. Neoh, Polym. Eng. Sci., 44, 362 (2004).

9. W. C. Wang, R. K. H. Vora, E. T. Kang, and K. G. Neoh, Macromol. Mater. Eng., 288, 152 (2003).

10. W. C. Wang, E. T. Kang, and K. G. Neoh, Appl. Surf. Sci., 199, 52 (2002).

11. W. H. Yu, Y. Zhang, E. T. Kang, K. G. Neoh, S. Y. Wu, and Y. F. Chow, J. Electrochem. Soc., 149, C521 (2002).

12. Z. J. Yu, E. T. Kang, and K. G. Neoh, Polymer, 43, 4137 (2002).

13. Y. Zhang, K. L. Tan, G. H. Yang, E. T. Kang, and K. G. Neoh, J. Electrochem. Soc., 148, C574 (2001).

14. G. H. Yang, E. T. Kang, K. G. Neoh, Y. Zhang, and K. L. Tan, Colloid Polym. Sci., 279, 745 (2001).

15. N. Inagaki, S. Tasaka, and T. Baba, J. Adhes. Sci. Technol., 15, 749 (2001).

16. N. Inagaki, S. Tasaka, and M. Masumoto, Macromolecules, 29, 1642 (1996).

17. N. Inagaki, S. Tasaka, H. Ohmori, and S. Mibu, J. Adhes. Sci. Technol., 10, 243 (1996).

18. N. Inagaki, S. Tasaka, and M. Masumoto, J. Appl. Polym. Sci., 56, 135 (1995).

19. N. Inagaki, S. Tasaka, and K. Hibi, J. Adhes. Sci. Technol., 8, 395 (1994).

20. K. Akamatsu, S. Ikeda, H. Nawafune, and H. Yanagimoto, J. Am. Chem. Soc., 126, 10822 (2004).

21. K. Akamatsu, S. Ikeda, and H. Nawafune, Langmuir, 19, 10366 (2003).

22. K. Akamatsu, S. Ikeda, H. Nawafune, and S. Deki, Chem. Mater., 15, 2488 (2003).

23. S. Ikeda, K. Akamatsu, and H. Nawafune, J. Mater. Chem., 
11, 2919 (2001).

24. T. H. Baum, D. C. Miller, and T. R. Otoole, Chem. Mater., 3, 714 (1991).

25. M. Mitsuishi, T. Tanuma, J. Matsui, J. F. Chen, and T. Miyashita, Langmuir, 17, 7449 (2001).

26. M. Mitsuishi, T. Tanuma, J. Matsui, and T. Miyashita, Talanta, 65, 1091 (2005).

27. J. Matsui, M. Mitsuishi, and T. Miyashita, J. Phys. Chem. B, 106, 2468 (2002).

28. A. Aoki, M. Nakaya, and T. Miyashita, Chem. Lett., 25, 667 (1996).

29. A. Aoki, M. Nakaya, and T. Miyashita, Macromolecules, 31, 7321 (1998).

30. Y. Kado, M. Mitsuishi, and T. Miyashita, Adv. Mater., 17, 1857 (2005).

31. J. Matsui, M. Mitsuishi, A. Aoki, and T. Miyashita, Angew. Chem., Int. Ed., 42, 2272 (2003).

32. J. Matsui, M. Mitsuishi, A. Aoki, and T. Miyashita, J. Am. Chem. Soc., 126, 3708 (2004).

33. J. Matsui, S. Yoshida, T. Mikayama, A. Aoki, and T.
Miyashita, Langmuir, 21, 5343 (2005).

34. H. Tanaka, M. Mitsuishi, and T. Miyashita, Langmuir, 19, 3103 (2003).

35. H. Tanaka, M. Mitsuishi, and T. Miyashita, Chem. Lett., 34, 1246 (2005).

36. Y. Kado, A. Aoki, and T. Miyashita, J. Mater. Sci., 37, 4761 (2002).

37. T. Miyashita, M. Nakaya, and A. Aoki, Supramol. Sci., 5, 363 (1998).

38. T. Miyashita, M. Nakaya, and A. Aoki, Thin Solid Films, 329, 833 (1998).

39. K. C. Grabar, R. G. Freeman, M. B. Hommer, and M. J. Natan, Anal. Chem., 67, 735 (1995).

40. M. J. Hostetler, J. E. Wingate, C. J. Zhong, J. E. Harris, R. W. Vachet, M. R. Clark, J. D. Londono, S. J. Green, J. J. Stokes, G. D. Wignall, G. L. Glish, M. D. Porter, N. D. Evans, and R. W. Murray, Langmuir, 14, 17 (1998).

41. S. Sawada, Y. Masuda, P. X. Zhu, and K. Koumoto, Langmuir, 22, 332 (2006). 